\title{
An Investigation into Transfer Effect of Brain Cognitive Training Contents with Text Network Analysis
}

\author{
Hyeok-Min Lee ${ }^{1}$, Sung-Wook Shin ${ }^{2}$, Ho-Sang Moon ${ }^{3}$ and Sung-Taek Chung ${ }^{*}$ \\ ${ }^{1,2,4}$ Department of Computer Engineering, Korea Polytechnic University, Gyeonggi, \\ Republic of Korea \\ ${ }^{3}$ Department of Advanced Technology Fusion, Korea Polytechnic University, \\ Gyeonggi, Republic of Korea \\ 1dlgurals12@kpu.ac.kr, ${ }^{2}$ napalza@kpu.ac.kr, ${ }^{3} h o s a n g 0815 @ k p u . a c . k r$, \\ ${ }^{4}$ unitaek@kpu.ac.kr
}

\begin{abstract}
In this paper, we aimed to analyze the transfer effect between cognitive areas using Computerized Cognitive Training (CCT). As a way to achieve goals, pieces of literature that have the effect of improving cognitive functions using CCT for Mild Cognitive Impairment (MCI) were collected from four research databases and performed analysis of the centrality based on text networks. As a result, the comparative experimental studies that used computers and video games together as a training tool accounted for the most, and memory accounted for most of the cognitive domain targeted by the training, and a variety of contents were performed for it. The most frequent memory training method was $N$-Back and, the contents with the highest centrality index was the memory area, and video was the highest as a tool of intervention. In particular, memory content and attention content were more than double the different with 33 points and 16 points. However, index of closeness centrality was found to be derived relatively similar scores at 0.387, 0.381. It can be interpreted that the possibility of the transfer effect occurring from memory and attention areas since the training process between the two content is similar.
\end{abstract}

Keywords: Computerized cognitive training, Mild cognitive impairment, Network centrality, Text-mining

\section{Introduction}

Mild Cognitive Impairment (MCI) is generally caused by decreased cognitive abilities, such as memory, judgment, concentration, and attention, due to aging. It is reported that accurate and appropriate diagnosis and treatment can minimize the damage of the cognitive functions [1][2]. Since cognitive training is applicable to patients with no cognitive or functional difficulties, it is more appropriate for patients with MCI [3]. The goal of cognitive training is to improve the cognitive ability in a specific area through repetitive learning on designed technique and strategy [4]. The cognitive process is known to occur through the activation of neurons related to brain regions biologically activated during the calculation and processing of specific stimuli from the external environment. In recent years, the distributed and overlapping control

Article history:

Received (October 16, 2020), Review Result (November 19, 2020), Accepted (December 28, 2020) 
architecture in which each brain area that controls cognitive function is in overlapping structures rather than functioning separately and efficiently activates cognitive functions through interaction has been reported. Several studies are ongoing on improving cognitive function by using a variety of information technologies, including virtual reality, interactive video gaming, and mobile technology, as intervention manner for cognitive training [5]. Among these technologies, Computerized Cognitive Training (CCT) relies on computers or tablet PCs as the intervention means and thus differs from the conventional face-to-face cognitive training that uses paper and pencil [6]. CCT has the advantage of providing objective data collection and real-time feedback since the cost and time efficiency, accessibility, and training content and difficulties can be specified for each user [7][8]. Although studies have reported the trend of intervention manners and verifying the effectiveness of CCT through meta-analysis of existing studies [9][10][11][12], there are no studies to-date on the analysis of transfer effect of CCT to other cognitive domains. For example, the study by Hill, Nicole TM, et al. (2017) [13] on the effectiveness of CCT discussed the meta-analysis and contents used for each of the various cognitive domains but did not analyze the association between the cognitive domains. Moreover, it has been reported that CCT is not effective on dementia patients, but there is no report that included the relevant data and reason. In another study, Zając-Lamparska, Ludmiła, et al. (2019) [14] performed meta-analysis on the efficacy of cognitive mediation using virtual reality but limited the intervention manner to virtual reality to possibly constrain the scope of studying CCT.

The amount of transfer effects in CCT could be determined by the pattern of training (e.g., training duration and intensity), participants' underlying cognitive abilities, supervision of training, and intrinsic motivation [15]. Furthermore, differences in the efficacy of that might be due to heterogeneity of methodological and structural between researches, and be related to standardized cognitive assessments used to evaluate training results [15]. In particular, adaptive training, which means training difficulty adjustment and continuous feedback for individual performance levels, had been shown to increase participants' motivation and produce greater transfer effects than non-adaptive training [17]. Thus, the more complexity, adaptability, and difficulty of cognitive training can improve the better cognitive ability of subjects' possibility of achieving transfer effects to untrained features.

In this study, we limited participants to patients with MCI based on the study results showing that CCT was not effective in dementia patients. However, it did not limit the computerized tools used by studies to analyze computer tools' diversity. Literatures published until December 2019 were collected and analyzed for trends by year, journal characteristics, and national trends of the main author and examined the transfer effect of computerized cognitive training contents by using a text network algorithm. While performing cognitive training, the patient trains one or two specific cognitive domains, and there is a possibility of transfer effects in which the cognitive ability of untrained areas is improved due to the adaptive manner [18]. Therefore, this study aimed to analyze the overall trend of CCT and whether the content of CCT targeting a specific cognitive domain is effective in other cognitive areas due to the transfer effect.

\section{Methods and materials}

\subsection{Searching strategy}

This study used four databases, viz. PubMed (NCBI), EMBASE, Cochrane Library, and Web of Science, to gather literature and analyzed the published study results up to December 2019. The literature collection used the search strategy by four types: study type, participant 
type, intervention type, and outcome type. Three researchers independently reviewed and discussed the collected papers based on whether they should be included in or excluded from this study, and the contents for the study were extracted accordingly.

\subsection{Inclusion \& Exclusion Criteria}

Studies: Only papers written in English were included, and those written in other languages were excluded. There was no limit in clinical trial design methods, and the studies on the development and design of CCT contents were excluded. Studies comparing the evaluation scores before and after CCT of MCI patients were included. Cases in which the clinical trial results were insufficient (i.e., the evaluation scores before and after computerized cognitive training being similar or decreased) were excluded, and only cases in which the results improved in more than $50 \%$ of the participants were included. Moreover, papers of which the full-text could not be collected were excluded.

Participants: The present study included papers that tested patients of age 60 years or older and diagnosed MCI based on Petersen's diagnosis criteria [19]. All papers focused on people with mental or neurological disorders other than MCI were not considered. Additionally, the papers that did not present data on MCI patients or subjected patients who may have dementia were excluded. Studies of a sufficient number of participants were included to derive the statistically significant results, and only the studies of 10 or more participants were included [20].

Interventions: The present study put no restriction on the means of CCT, e.g., virtual reality, video game, computer, and tablet PC. However, it excluded the papers that used CCT and nonCCT (training using pencil and paper) since the outcomes can be confusing. Furthermore, papers that did not provide a detailed description of the contents of CCT through discussion were computerized, while the papers that did not explicitly state the targeted cognitive domain were excluded.

Outcomes: We included literature on training the memory, attention, and executive function domains based on the five major cognitive domains (orientation, memory, attention, executive function, and language) evaluated in the Mini Mental State Examination (MMSE) [21]. Since orientation was a sub-item of attention and memory, it was included as attention and memory domains, and the language domain was excluded because it can be less effective in judging MCI due to differences in language used in each country [22][23]. Moreover, since orientation to time, orientation to place, calculation, recall, copying, and 3-step command among the MMSE subcategories are related to activities of daily living (ADL), we included the studies aiming at training in the four cognitive domains: memory, attention, executive function, and ADL [24].

\subsection{Keywords \& study selection}

A total of 888 papers were identified in four databases after the initial literature search, and three additional papers were collected by manually searching for the references in the collected papers related to this study in Google Scholar. Through the literature identification process, the duplicate papers were removed, while through the eligibility process the subject and abstract were reviewed and those that did not meet the inclusion criteria were removed. In this way, 82 papers were selected via the eligibility process. Those 82 studies used CCT in the treatment of MCI patients. The inclusion process reviewed the main text and removed the papers that did not meet the criteria of the study type, participant type, intervention type, and outcome type. 
The final 18 papers selected in the inclusion process signified that they used CCT to treat MCI patients and showed positive clinical outcomes.

\subsection{Text network analysis in $R$}

It performed the text network analysis using Quanteda package of R (version 3.6.2) statistical software to investigate the transfer effect of CCT. The present study calculated the Degree Centrality (DC), Closeness Centrality (CC), and Betweenness Centrality (BC) to analyze the link strength between the keywords. The DC refers to the number of keywords linked with a keyword. A higher centrality indicates that the keyword is linked with many other keywords. The calculation for the DC can be expressed as Equation 1 [25]:

$$
C_{d}\left(N_{i}\right)=\sum_{j=1}^{k-1} x_{i j} \quad(i \neq j)
$$

where $\mathrm{C}_{\mathrm{d}}\left(\mathrm{N}_{\mathrm{i}}\right)$ refers to the DC of the $i^{\text {th }}$ keyword $N, k$ refers to the total number of keywords, $x_{i j}$ denotes an index representing the total number of keywords where the $i^{\text {th }}$ keyword has a connection with $k-1$ keywords except the $i^{\text {th }}$ one. To note, the DC only shows the frequency of connection between the keywords; therefore, it becomes necessary to analyze the CC. The CC calculates the direct connections between the keywords and indirect connections to calculate the distance between a keyword and all other keywords to extract the influential keywords. In other words, the index represents how close a keyword is with another keyword. Therefore, a higher CC signifies a higher possibility of diffusion to the connected keyword. For example, we can infer that a higher CC between the memory and attention domains means that the CCT contents for memory training can be effective for attention training as well. The calculation for the $\mathrm{CC}$ can be expressed as Equation 2 [26]:

$$
C_{c}\left(N_{i}\right)=\frac{1}{\sum_{j=1}^{k} d\left(N_{i}, N_{j}\right)}
$$

where $\mathrm{C}_{\mathrm{c}}\left(\mathrm{N}_{\mathrm{i}}\right)$ refers to the $\mathrm{CC}$ of the $i^{\text {th }}$ keyword $N$, and $\mathrm{d}\left(\mathrm{N}_{\mathrm{i}}, \mathrm{N}_{\mathrm{j}}\right)$ refers to the shortest path between the $i^{\text {th }}$ and $j^{\text {th }}$ keywords. The present study visualized the DC and CC indices between the keywords via thickness of the line or link that connected two keywords. Therefore, two keywords which are closer or have a thicker line between them mean that they have a more frequent or influential relationship. Additionally, we calculated the $\mathrm{BC}$ to evaluate to what extent a keyword can play a mediating role in the connections between other keywords in the network. In this study's network of CCT contents, presence of a high BC means that the content can play a role in connecting other disconnected cognitive domains. In other words, it implied that the keyword in the middle position plays the role of a broker and connects other keywords. as the $\mathrm{BC}$ can be calculated using Equation 3 [27]:

$$
C_{b}\left(N_{i}\right)=\sum_{j<k} \frac{g_{j k}\left(N_{i}\right)}{g_{j k}}
$$

where $\mathrm{C}_{\mathrm{b}}\left(\mathrm{N}_{\mathrm{i}}\right)$ refers to the $\mathrm{BC}$ of the $i^{\text {th }}$ keyword $N$, $\mathrm{g}_{\mathrm{jk}}$ refers to the number of the shortest paths between the $j^{\text {th }}$ and $k^{\text {th }}$ keywords, and $\mathrm{g}_{\mathrm{jk}}\left(\mathrm{N}_{\mathrm{i}}\right)$ refers to the number of paths that include the keyword $\mathrm{N}_{\mathrm{i}}$ between the $j^{\text {th }}$ and $k^{\text {th }}$ keywords. The $\mathrm{BC}$ represents the extent to which a keyword (playing the role of a broker) lies in the shortest path between two other keywords and indicates the number of keywords located in the path in a network. The current work developed and analyzed a network by connecting all the keywords used in the intervention manners and contents in a specific cognitive domain based on the co-occurrence matrix, which expressed 
frequencies of the keywords in an array using all indices described above. We also categorized the CCT contents which are widely used in a specific cognitive domain based on it and analyzed how effective the contents are in a cognitive domain.

\section{Results}

In the 18 papers that reported the effectiveness of clinical trials, computer was the most widely used tool for CCT, while video and virtual reality were used as the medium. The comparative experimental studies that used computers and video games together as a training tool accounted for the most, and Nintendo and iPad were used as the media of the video game. All studies stated the process of computerized training to the participants and provided sufficient education and training. The training contents were either commercially available or designed specifically matched with the study objective. Only the studies that reported significant effect of training were included. Memory accounted for most of the cognitive domain targeted by the training, and a variety of contents were performed for it. The most frequent memory training method used by the 18 studies was N-Back, and each study designed their contents based on the different memory areas, viz. occupational memory, short-term memory, and instantaneous memory. Since the lower-level recognition domains were divided according to the cognitive characteristics of the higher-level cognitive domains, the analysis included them in the higher-level cognitive domains of the target cognitive area [28]. The training period varied widely between 1 week and 26 weeks, and most studies compared the participant groups and observed the effect after training of one month or longer. The training on activities of daily living involved activities such as grocery shopping, cooking, and washing, which are routine activities of daily life. All studies performed them in a virtual reality environment. The training was conducted with one or two target cognitive areas to each content, and the training period varied widely between 1 week and 12 weeks.

Table 1 . Top 10 centrality of keywords

\begin{tabular}{|c|c|c|c|c|c|c|}
\hline Rank & Keywords & DC & Keywords & CC & Keywords & BC \\
\hline 1 & CCT_Contents & 61 & CCT_Contents & 1.053 & Memory_Contents & 64 \\
\hline 2 & Memory_Contents & 33 & Memory_Contents & 0.387 & Attention_Contents & 13 \\
\hline 3 & TCA & 23 & Attention_Contents & 0.381 & Video & 6 \\
\hline 4 & Type_of_CCT & 18 & ADL_Contents & 0.254 & ADL_Contents & 5 \\
\hline 5 & Attention_Contents & 16 & IT & 0.223 & Executive_Contents & 3 \\
\hline 6 & Memory & 8 & TCA & 0.223 & Computer & 0 \\
\hline 7 & ADL_Contents & 5 & Executive_Contents & 0.22 & VR & 0 \\
\hline 8 & Video & 3 & Computer & 0.216 & Nintendo & 0 \\
\hline 9 & Executive_Contents & 3 & VR & 0.216 & Ipad & 0 \\
\hline 10 & \multicolumn{2}{|c|}{ Computer } & 2 & Video & 0.216 & \\
\hline
\end{tabular}

[Table 1] lists the calculation results of the centrality indices. In the DC index, memory was a CCT content having the highest frequency 33, followed by attention with frequency 16, activities in daily living with frequency 5 , and executive ability with frequency 3 . Likewise, memory accounted as the largest training target domain with frequency 14 , followed by 
attention with frequency 7 , executive ability with frequency 2 , and activities in daily living with frequency 1 . The indices of the memory contents and the attention contents were relatively high in $\mathrm{CC}$ index, indicating that the studies focused more on memory and attention than executive ability and activities of daily living. Although the frequency of memory training contents was more than twice that of the attention contents when analyzing only the DC, they showed similar scores of 0.387 and 0.381 , respectively, in the CC index. It means that the semantic distance between the memory training contents and attention training contents is short and implied that performing memory training would be effective for the attention domain also. CoTras, which is the training contents targeting memory, is sometimes performed also for attention. However, it is segmented for training of each area and has not been analyzed for transfer effects. In the $\mathrm{BC}$ index, the memory training contents were the highest with frequency 64 , indicating that it is likely to be used more widely. The BC increases as there are more target cognitive domains of the collected studies and is equal to the number of computerized cognitive training contents if there is only one domain. Moreover, the BC index of the video was higher than the other intervention manners, indicating that many studies tried to verify the effectiveness by comparing video with other intervention manners.

\section{Discussion and conclusions}

CCT is a method of improving cognitive ability, such as memory, attention, and executive ability, using digital media without intervention of any drugs [3]. It is used to improve the cognitive ability of MCI patients rather than the dementia patients. Most contents of CCT are designed with the expectation of improvement in the cognitive domain targeted by the study [4]. However, there are no studies on the impact of training using the contents designed for the specific study objective on other untrained domain. To investigate the transfer effect of CCT, the current work collected papers that performed CCT and selected the ones that reported clinical effects of performing text network analysis. Out of 888 papers collected initially, 82 were found to study MCI patients' training for memory, attention, executive ability, and activities in daily living. Out of those 82 papers, 18 papers verified the effectiveness through clinical trials.

In the 18 selected papers that reported the clinical effect, the training targeting memory accounted for the most CCT contents with clinical effects. They used computers, video games, and virtual reality environments according to the study objective. Activities of daily living, such as washing, shopping, moving items, and eating, used virtual reality environments to emphasize reality. The studies were mostly designed for randomized controlled trials, and there was no significant statistical difference between the number of participants and gender. The 18 selected papers were investigated for keyword connection related to three-centrality analysis (DC, CC, and BC) and three types (CCT, intervention manner, and training target domain) in the text network. The DC index showed a difference of more than double as there were 33 contents for memory training and 16 contents for attention training, meaning that memory contents were studied more than attention contents. Moreover, it was confirmed that relatively similar scores in CC were derived for the memory and attention domains, 0.387 and 0.381 , respectively. It indicates that the two contents' training processes may be similar and that a transfer effect may appear between the two domains. The attention content generally receives a higher score as more items are processed simultaneously in a shorter period, and the memory content receives a higher score as more items are stored longer in the memory. Therefore, we can consider that the two domains are related because it is necessary to pay more attention to 
process the items simultaneously in a short period when more items are stored in the memory. In the $\mathrm{BC}(\mathrm{BC})$ index, the memory contents were the highest with frequency 64 , indicating that it is likely to be used more widely. The high $\mathrm{BC}$ index of the video game indicated that many studies compared it with other intervention manners (e.g., virtual reality and computer) to verify its effectiveness. However, one of the papers collected for our study reported that the content was effective on memory, but no further studies were done to prove its effectiveness on attention.

As a future work, it is necessary to study on contents that are the most effective in each cognitive domain through effect size analysis of the collected papers, and more samples should be collected to study on domains that showed the transfer effect. They can verify the effectiveness of CCT for treating MCI without using any drug.

\section{Acknowledgements}

This work was supported by the National Research Foundation of Korea (NRF2020R1A2C1011960) grant funded by the Korea government (MSIT), the MSIT (Ministry of Science and ICT), Korea, under the ITRC (Information Technology Research Center) support program (IITP-2020-2018-0-01426) supervised by the IITP (Institute for Information \& Communications Technology Planning \& Evaluation).

\section{References}

[1] A. M. Sanford, "Mild cognitive impairment," Clinics in Geriatric Medicine, vol.33, no.3, pp.325-337, (2017) DOI: 10.1016/j.cger.2017.02.005

[2] D. S. Knopman, and R. C. Petersen, "Mild cognitive impairment and mild dementia: A clinical perspective," Mayo Clinic Proceedings, vol.89, no.10, pp.1452-1459, (2014) DOI: 10.1201/b13196-57

[3] H. Zhang, J. Huntley, R. Bhome, B. Holmes, and J. Cahill, et. al, "Effect of computerized cognitive training on cognitive outcomes in mild cognitive impairment: A systematic review and meta-analysis," BMJ open, vol.9, no.8, (2019) DOI: 10.1136/bmjopen-2018-027062

[4] N. J. Gates and M. F. Singh, "Exercise and cognitive function in older adults," Diet and Exercise in Cognitive Function and Neurological Diseases, pp.279-294, (2015) DOI: 10.1002/9781118840634.ch24

[5] S. Ge, Z. Zhu, B. Wu, and E. S. McConnell, "Technology-based cognitive training and rehabilitation interventions for individuals with mild cognitive impairment: A systematic review," BMC Geriatrics, vol.18, no.1, (2018) DOI: 10.1186/s12877-018-0893-1

[6] N. Gates and M. Valenzuela, "Cognitive exercise and its role in cognitive function in older adults," Current Psychiatry Reports, vol.12, no.1, pp.20-27, (2010) DOI: 10.1007/s11920-009-0085-y

[7] M. J. Chandler, A. C. Parks, M. Marsiske, L. J. Rotblatt, and G. E. Smith, "Everyday impact of cognitive interventions in mild cognitive impairment: A systematic review and meta-analysis," Neuropsychology Review, vol.26, no.3, pp.225-251, (2016) DOI: 10.1007/s11065-016-9330-4

[8] H. Coyle, V. Traynor, and N. Solowij, "Computerized and virtual reality cognitive training for individuals at high risk of cognitive decline: Systematic review of the literature,” The American Journal of Geriatric Psychiatry, vol.23, no.4, pp.335-359, (2015) DOI: 10.1016/j.jagp.2014.04.009

[9] Bahar-Fuchs, Alex, Linda Clare, and Bob Woods, "Cognitive training and cognitive rehabilitation for persons with mild to moderate dementia of the Alzheimer's or vascular type: A review," Alzheimer's Research and Therapy, vol.5, no.4, (2013) DOI: 10.1186/alzrt189

[10] L. Jean, M.-È. Bergeron, S. Thivierge, and M. Simard, "Cognitive intervention programs for individuals with mild cognitive impairment: Systematic review of the literature," The American Journal of Geriatric Psychiatry, vol.18, no.4, pp.281-296, (2010) DOI: 10.1097/jgp.0b013e3181c37ce9 
[11] J. Reijnders, C. Van Heugten, and M. van Boxtel, "Cognitive interventions in healthy older adults and people with mild cognitive impairment: A systematic review," Ageing Research Reviews, vol.12, no.1, pp.263-275, (2013) DOI: 10.1016/j.arr.2012.07.003

[12] M.J. Chandler, A.C. Parks, M. Marsiske, L.J. Rotblatt, and G.E. Smith, "Everyday impact of cognitive interventions in mild cognitive impairment: A systematic review and meta-analysis," Neuropsychology Review, vol.26, no.3, pp.225-251, (2016) DOI: 10.1007/s11065-016-9330-4

[13] N.T.M. Hill, L. Mowszowski, S.L. Naismith, V.L. Chadwick, M. Valenzuela, and A. Lampit, "Computerized cognitive training in older adults with mild cognitive impairment or dementia: A systematic review and meta-analysis," American Journal of Psychiatry, vol.174, no.4, pp.329-340, (2017) DOI: 10.1176/appi.ajp.2016.16030360

[14] L. Zając-Lamparska, M. Wiłkość-Dębczyńska, A. Wojciechowski, M. Podhorecka, A. Polak-Szabela, Ł. Warchoł, K. Kędziora-Kornatowska, A. Araszkiewicz, and P. Izdebski, "Effects of virtual realitybased cognitive training in older adults living without and with mild dementia: A pretest-posttest design pilot study,” BMC Research Notes, vol.12, no.1, (2019) DOI: 10.1186/s13104-019-4810-2

[15] A. Lampit, H. Hallock, and M. Valenzuela, "Computerized cognitive training in cognitively healthy older adults: a systematic review and meta-analysis of effect modifiers," PLoS medicine, vol.11, no.11, e1001756, (2014) DOI: 10.1371/journal.pmed.1001756

[16] P.D. Gajewski, S. Thönes, M. Falkenstein, E. Wascher, and S. Getzmann, "Multidomain cognitive training transfers to attentional and executive functions in healthy older adults," Frontiers in Human Neuroscience, vol.14, e586963, (2020) DOI: 10.3389/fnhum.2020.586963

[17] J. Mishra, E. de Villers-Sidani, M. Merzenich, and A. Gazzaley, "Adaptive training diminishes distractibility in aging across species," Neuron, vol.84, no.5, pp.1091-1103, (2014) DOI: 10.1016/j.neuron.2014.10.034

[18] M.J. Chandler, A.C. Parks, M. Marsiske, L.J. Rotblatt, and G. E. Smith, "Everyday impact of cognitive interventions in mild cognitive impairment: A systematic review and meta-analysis," Neuropsychology Review, vol.26, no.3, pp.225-251, (2016) DOI: 10.1007/s11065-016-9330-4

[19] R.C. Petersen, G.E. Smith, S.C. Waring, and R.J. Ivnik, et al, "Mild cognitive impairment: Clinical characterization and outcome," Archives of neurology, vol.56, no.3, pp.303-308, (1999)

[20] D. Machin, M.J. Campbell, S.B. Tan, and S.H. Tan, "Sample sizes for clinical, laboratory and epidemiology studies," John Wiley and Sons, (2018)

[21] K. Shigemori, S. Ohgi, E. Okuyama, T. Shimura, and E. Schneider, "The factorial structure of the mini mental state examination (MMSE) in Japanese dementia patients," BMC Geriatrics, vol.10, no.1, (2010) DOI: $10.1186 / 1471-2318-10-36$

[22] M.D. Lezak, D.B. Howieson, D.W. Loring, and J.S. Fischer, "Neuropsychological assessment," Oxford University Press, (2004)

[23] Y.S. Shim, D.W. Yang, H.-J. Kim, Y.H. Park, and S. Kim, "Characteristic differences in the minimental state examination used in Asian countries," BMC Neurology, vol.17, no.1, (2017) DOI: 10.1186/s12883-017-0925-z

[24] G. Han, M. Maruta, Y. Ikeda, T. Ishikawa, H. Tanaka, A. Koyama, R. Fukuhara, S. Boku, M. Takebayashi, and T. Tabira, "Relationship between performance on the mini-mental State examination sub-items and activities of daily living in patients with Alzheimer's disease," Journal of Clinical Medicine, vol.9, no.5, p.1537, (2020) DOI: 10.3390/jcm9051537

[25] J. Nieminen, "On the centrality in a graph," Scandinavian journal of psychology, vol.15, no.1, pp.332 336, (1974)

[26] S. Wasserman, and K. Faust, "Social network analysis: Methods and applications," Cambridge University press, vol. 8, (1994)

[27] L. Freeman, “Centrality in networks: I. conceptual clarifications. Social networks,” pp.215-239, (1979) 
[28] J. Lehrner, J. Maly, A. Gleiss, E. Auff, et. al, "Neuropsychological performance and mild cognitive impairment subtypes in patients reporting cognitive problems attending a memory outpatient clinic," European Journal of Geriatrics, vol.10, no.2 pp.59-68, (2008) 
This page is empty by intention. 